\title{
Fragilidades e potencialidades da equipe multiprofissional no desenvolvimento dos cuidados paliativos em Unidade de Terapia Intensiva
}

\author{
Weaknesses and strengths of the multidisciplinary team in the development of palliative care in an
}

Intensive Care Unit

Debilidades y fortalezas del equipo multidisciplinario en el desarrollo de cuidados paliativos en la Unidad de Cuidados Intensivos

\section{Resumo}

Os cuidados paliativos (CP) consistem na assistência promovida por equipe multiprofissional, que objetiva a melhoria da qualidade de vida do paciente e de seus familiares diante de uma doença que ameace a vida. Assim, foi realizada uma revisão integrativa com objetivo de identificar na literatura as fragilidades e potencialidades da equipe multidisciplinar no desenvolvimento dos cuidados paliativos em Unidade de Terapia Intensiva (UTI). A busca de artigos científicos foi realizada nas bases de dados Google Acadêmico, PubMed e Biblioteca Virtual em Saúde em julho de 2020, sendo selecionados 11 artigos para compor a revisão. Observou-se que as potencialidades ainda são pouco reconhecidas pela maioria dos profissionais que fazem parte da equipe multiprofissional dentro das UTI, sendo que estes reconhecem a necessidade de oferecer conforto nos momentos finais de vida e percebem que a presença de CP na UTI pode melhorar a qualidade do paciente em fim de vida. Porém, ainda são observadas muitas fragilidades/dificuldades para implementação desses cuidados, como: trabalho não compartilhado em equipe, falta de interação e comunicação entre profissionais da equipe multidisciplinar; despreparo dos profissionais para lidar com o paciente em CP devido à falta de conhecimento e formação adequada, conflitos éticos e ausência de protocolos específicos. É importante oferecer treinamento e educação continuada para capacitação desses profissionais para os CP e é necessário implantar protocolos específicos em cuidados paliativos nos ambientes de terapia intensiva, para a redução do sofrimento e para a melhora da qualidade do atendimento oferecido a este paciente em fase final de vida.

Palavras-chave: Cuidados de conforto; Unidade de Terapia Intensiva; Paliativismo; Interdisciplinaridade.

\begin{abstract}
Palliative care (PC) consists of assistance provided by a multidisciplinary team, which aims to improve the quality of life of patients and their families in the face of a life-threatening disease. Thus, an integrative review was carried out in order to identify in the literature the weaknesses and strengths of the multidisciplinary team in the development of palliative care in the Intensive Care Unit (ICU). The search for scientific articles was performed in the Google Academic, PubMed and Virtual Health Library databases in July 2020, with 11 articles being selected to compose the review. It was observed that the potentials are poorly recognized by most professionals who are part of the multidisciplinary team within the ICU, and they recognize the need to offer comfort in the final moments of life and realize that the presence of PC in the ICU can improve the end-of-life patient quality. However, there are still many weaknesses/difficulties in implementing this care, such as: non-shared team work, lack of interaction and communication between professionals in the multidisciplinary team, the unpreparedness of professionals to deal with patients in PC due to lack of knowledge and adequate training, and also, ethical conflicts and the absence of specific protocols. It is important to offer training and continuing education to qualify these professionals for PC and it is necessary to implement specific protocols in palliative care in intensive care settings, to reduce suffering and to improve the quality of care offered to this patient in the final phase of life.
\end{abstract}

Keywords: Comfort care; Intensive Care Unit; Palliative care; Interdisciplinarity. 


\begin{abstract}
Resumen
Los cuidados paliativos (CP) consisten en la asistencia brindada por un equipo multidisciplinar, que tiene como objetivo mejorar la calidad de vida de los pacientes y sus familias ante una enfermedad mortal. Así, se realizó una revisión integradora con el fin de identificar en la literatura las debilidades y fortalezas del equipo multidisciplinario en el desarrollo de los CP en la Unidad de Cuidados Intensivos (UCI). La búsqueda de artículos científicos se realizó en las bases Google Academic, PubMed y Biblioteca Virtual de Salud en julio de 2020, seleccionándose 11 artículos para la revisión. Se observó que las potencialidades son poco reconocidas por la mayoría de los profesionales del equipo multidisciplinario dentro de la UCI, y reconocen la necesidad de ofrecer comodidad en los momentos finales de la vida y que la presencia de CP en la UCI puede mejorar. Sin embargo, aún existen muchas debilidades en la implementación de esta atención, como: el trabajo en equipo no compartido, la falta de interacción y comunicación entre los profesionales del equipo multidisciplinar, la falta de preparación de los profesionales para el tratamiento de los pacientes en AP por falta de conocimiento y formación adecuada, y también, conflictos éticos y ausencia de protocolos específicos. Es importante ofrecer formación y educación continua para capacitar a estos profesionales en AP y es necesario implementar protocolos específicos en cuidados paliativos en cuidados intensivos, para reducir el sufrimiento y mejorar la calidad de la atención ofrecida a este paciente en la fase final de la vida.
\end{abstract}

Palabras clave: Cuidado de la comodidad; Unidad de Cuidados Intensivos; Cuidados paliativos; Interdisciplinariedad.

\title{
1. Introdução
}

Com o passar dos tempos, a morte deixou de ser vivenciada no lar, passando a ser institucionalizada. No mundo atual a maioria dos óbitos ocorre nos hospitais e, mais especificamente, nas Unidades de Terapia Intensiva (UTI) (Gulini et al., 2017). É incontestável que a expectativa de vida tem aumentado e que a medicina moderna tem permitido o tratamento e a cura de inúmeras doenças (Silva et al., 2013). Entretanto, em muitas ocasiões, a tecnologia tem levado ao prolongamento do morrer (Moritz et al., 2009).

Hoje é difícil reconhecer e aceitar a finitude, inclusive para profissionais da saúde, que muitas vezes recorrem a medidas desproporcionais para evitar o fim da vida, prolongando o sofrimento, no lugar de ações paliativas (Junior et al., 2019).

Os cuidados paliativos (CP) consistem na assistência promovida por uma equipe multidisciplinar, que objetiva a melhoria da qualidade de vida do paciente e de seus familiares diante de uma doença que ameace a vida, por meio da prevenção e do alívio do sofrimento, da identificação precoce, avaliação impecável e tratamento de dor e demais sintomas físicos, sociais, psicológicos e espirituais (Baptista \& Picanço, 2019).

A Organização Mundial de Saúde (OMS, 2018) concluiu que apenas 14\% daqueles que têm indicação de tratamento paliativo o recebem. Esse tipo de assistência visa preencher a lacuna entre conhecimento científico e humanístico, buscando resgatar a dignidade da vida e a possibilidade de morrer como se deseja (Maingué et al., 2020).

O CP se apoia na visão da ortotanásia, que se caracteriza pela morte em seu tempo natural, garantindo a dignidade do indivíduo e promovendo o seu bem estar, com a finalidade de produzir uma "boa morte" (Silva et al., 2013). Um estudo demonstrou que na percepção dos profissionais, a prática que visa respeito à dignidade humana por meio de morte digna, além de estar envolta em princípios bioéticos que permitem suspender medidas curativas, estão dando espaço para o paliativismo como mecanismo de diminuição do sofrimento e da dor nesses pacientes (Santana et al., 2017).

A investigação na área dos CP tem contribuído para a sua difusão e implementação, com um papel fundamental no cumprimento dos seus princípios, filosofia e objetivos (Souza et al., 2017). Dessa forma, o CP vem ocupando lugar de destaque nas discussões atuais dos profissionais da área da saúde, na assistência a pacientes com doenças crônicas sem possibilidade de cura e, mais recentemente, a pacientes em situação aguda, como os internados em UTI (Gulini et al., 2017).

No tratamento paliativo, a interdisciplinaridade é absolutamente necessária. O plano de cuidados e o planejamento terapêutico devem envolver toda a equipe, buscando sempre melhorar a qualidade de vida do enfermo e de seus familiares 
(PIRES et al., 2020). Além da competência científica, a formação dos profissionais deve incluir a bioética e as humanidades. E para promover o acolhimento e o cuidado, a atualização frequente é sempre muito importante(Santos, 2011).

A participação efetiva da equipe multiprofissional ganha espaço em diferentes estágios dos $\mathrm{CP}$, desde sua indicação, alcançando a conferência familiar e se consolidando com as ações de cuidado e conforto propriamente ditas (Silva et al., 2013). Por outro lado, não é difícil que integrantes da equipe de saúde não reconheçam nos CP um espaço especial de cuidado. As ações na esfera paliativa, juntamente com a ação curativa, são tidas como conflituosas e desafiadoras para muitos profissionais da saúde (Junior et al., 2019).

Tendo em vista que a formação acadêmica da equipe multiprofissional é voltada para salvar vidas e prestar um cuidado curativo, a abordagem para o paliativismo permanece pouco explorada (Pegoraro \& paganini, 2019). Além da formação muitas vezes incipiente nessa temática, para o profissional da saúde é difícil a percepção de que sua assistência não deu o resultado esperado, o que torna necessária a abordagem paliativa. Por isso, a reflexão e a discussão da visão paliativista nos ambientes hospitalares ainda são desafiadoras (Maingué et al., 2020).

Além disso, há uma escassez de publicações que avaliem o paliativismo sob a ótica dos diferentes profissionais que constituem a equipe multiprofissional que assistem ao paciente grave em fase final da vida (Santos et al., 2017). Grande parte dos estudos estão direcionados à filosofia do CP ou se restringem a trabalhos envolvendo uma classe profissional específica, se distanciando da proposta da integralidade no cuidado a esse perfil de paciente (Vicensi, 2016).

Nesse contexto, este estudo teve por objetivo identificar na literatura científica as fragilidades e potencialidades da equipe multidisciplinar no desenvolvimento dos cuidados paliativos em UTI.

\section{Metodologia}

Foi realizada uma revisão integrativa com objetivo de identificar na literatura as fragilidades e potencialidades da equipe multidisciplinar no desenvolvimento dos cuidados paliativos (CP) em Unidade de Terapia Intensiva (UTI). Essa modalidade de revisão utiliza uma metodologia abrangente, que possibilita a análise de estudos com diferentes desenhos de pesquisa, de natureza quantitativa ou qualitativa, e abordagens experimentais e não-experimentais (Souza et al., 2010). Os procedimentos metodológicos adotados foram: formulação da questão e dos objetivos da revisão; definição e aplicação dos critérios de seleção de artigos; categorização dos estudos; análise dos dados e interpretação dos resultados; e síntese dos achados da revisão.

A busca de artigos científicos foi realizada nas bases de dados Google Acadêmico, PubMed e Biblioteca Virtual em Saúde (BVS), em julho de 2020, utilizando os descritores em português: "Cuidados paliativos" AND "Unidade de Terapia Intensiva" AND "Equipe de Assistência ao Paciente"; e seus correspondentes em inglês ("Palliative Care" AND "Intensive Care Units" AND "Patient Care Team") e em espanhol ("Cuidados paliativos" AND "Unidade de Cuidados Intensivos" AND "Equipe de Assistência ao Paciente"), identificados no título e/ou resumo.

Foram considerados como critérios de inclusão artigos científicos que continham os descritores listados neste protocolo e publicados em periódicos científicos de livre acesso na forma completa, nos idiomas português, inglês e espanhol, e que identificassem fragilidades e potencialidades envolvendo a equipe multiprofissional em cuidados paliativos em UTI adulto. Foram excluídos: editoriais, cartas, artigos de opinião e de revisão, comentários, resumos de anais, ensaios, publicações duplicadas, teses, dissertações, trabalhos de conclusão de cursos, boletins epidemiológicos, relatórios de gestão, documentos oficiais de programas nacionais e internacionais, livros e materiais publicados em outros idiomas que não sejam os do item de inclusão, bem como, estudos que não contemplem o escopo definido neste protocolo.

Para a primeira seleção foram lidos os títulos e resumos dos artigos, por três revisores independentes. Os artigos selecionados foram lidos na integra observando se de fato atendiam aos critérios da pesquisa. Os artigos selecionados para 
revisão, foram analisados e sistematizados por autoria, ano de publicação, tipo de estudo, objetivo principal, fragilidades e potencialidades encontradas e principais conclusões.

\section{Resultados}

Foram encontrados um total de 687 estudos por meio da busca nas bases de dados utilizando os descritores citados anteriormente, sendo estes 170 no Google Acadêmico, 83 na PubMed e 425 no Portal BVS.

Como mostra a Figura 1, 213 artigos foram excluídos por estarem duplicados, restando 474 títulos e resumos para leitura. Destes, 451 foram excluídos por não serem artigos científicos, ou serem estudos realizados em UTI neonatal ou não estar disponível de forma completa. Os 23 artigos potencialmente elegíveis foram lidos na íntegra, observando se de fato atendiam os critérios estabelecidos. Assim, mais 12 artigos foram excluídos (foram encontrados artigos de revisão e outros que focavam somente na visão de um profissional e não da equipe multiprofissional), totalizando 11 artigos selecionados para essa revisão.

Figura 1. Diagrama dos estudos selecionados no processo de revisão sistemática.

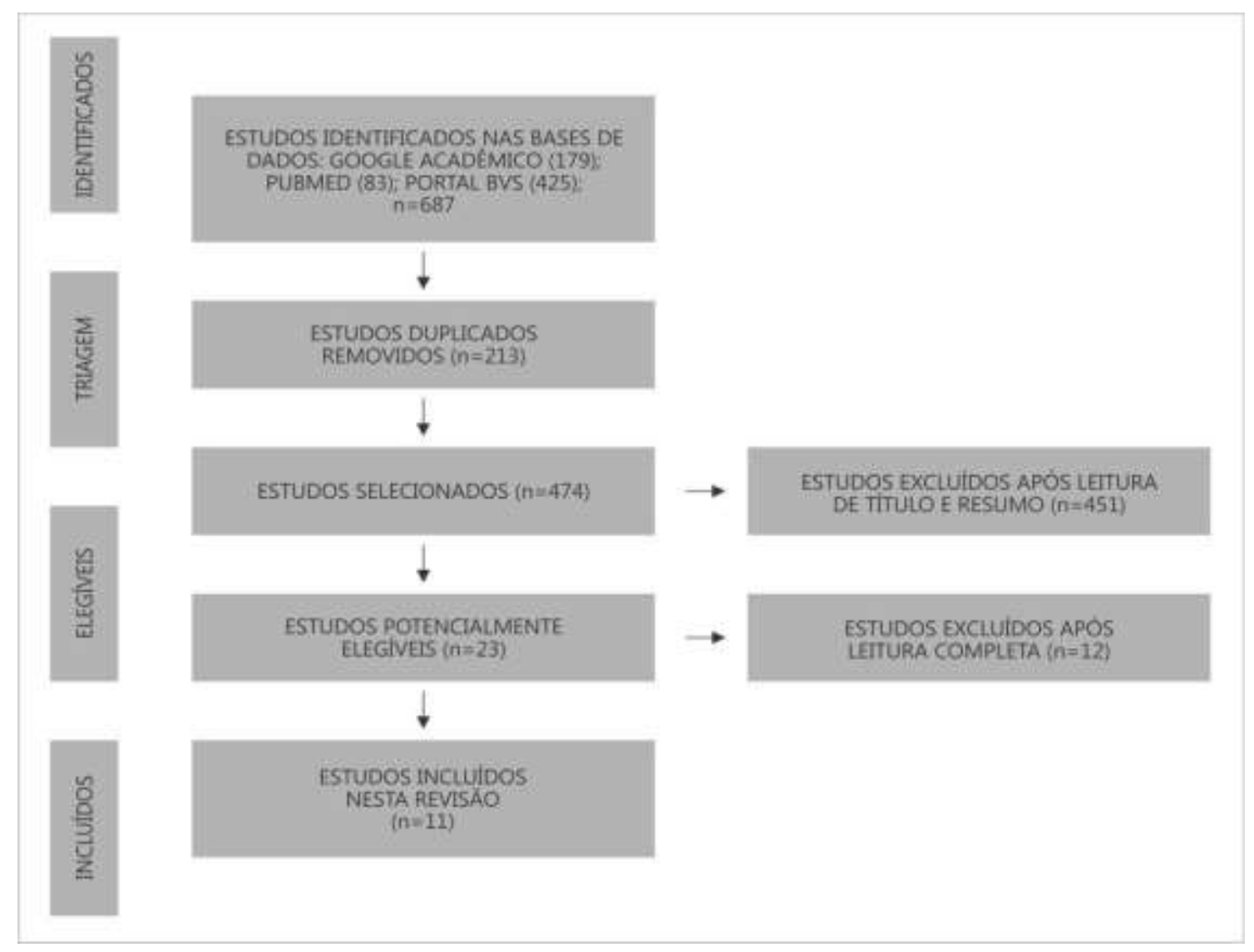

Fonte: Autoras (2020).

No Quadro 1 estão sumarizadas as informações obtidas dos artigos sobre as fragilidades e potencialidades da equipe multidisciplinar no desenvolvimento dos cuidados paliativos em Unidade de Terapia Intensiva (UTI). Quadro 1. Estudos que versam sobre as fragilidades e potencialidades da equipe multidisciplinar no desenvolvimento dos cuidados paliativos (CP) em Unidade de Terapia Intensiva (UTI). 
Quadro 1. Artigos selecionados para a pesquisa.

\begin{tabular}{|c|c|c|c|c|c|}
\hline $\begin{array}{c}\text { Autores } \\
\text { (Ano) }\end{array}$ & $\begin{array}{l}\text { Tipo de } \\
\text { estudo }\end{array}$ & Objetivo principal & Fragilidades encontradas & $\begin{array}{c}\text { Potencialidades } \\
\text { encontradas }\end{array}$ & Principais Conclusões \\
\hline $\begin{array}{l}\text { Silva et al. } \\
\quad \text { (2013) }\end{array}$ & $\begin{array}{l}\text { Pesquisa } \\
\text { exploratória, } \\
\text { descritiva, } \\
\text { qualitativa. }\end{array}$ & $\begin{array}{l}\text { Analisar as } \\
\text { concepções da } \\
\text { equipe } \\
\text { multiprofissional } \\
\text { sobre a } \\
\text { implementação de } \\
\text { CP em uma UTI } \\
\text { adulto. }\end{array}$ & $\begin{array}{l}\text { - Profissionais conhecem } \\
\text { parcialmente a proposta dos } \\
\text { CP; } \\
\text { - Foram observadas } \\
\text { divergências nas condutas } \\
\text { terapêuticas desenvolvidas pela } \\
\text { equipe durante a prática } \\
\text { assistencial; } \\
\text { - Observou-se falta de interação } \\
\text { e comunicação entre os } \\
\text { profissionais da equipe } \\
\text { multidisciplinar. }\end{array}$ & $\begin{array}{l}\text { - Na assistência ao } \\
\text { paciente em fase final } \\
\text { de vida, a importância } \\
\text { de priorizar o conforto } \\
\text { foi destacada pelos } \\
\text { entrevistados, que } \\
\text { enfatizaram o controle } \\
\text { da dor; } \\
\text {-Realização de } \\
\text { discussões dos casos } \\
\text { clínicos entre a equipe } \\
\text { multidisciplinar, } \\
\text { mediada pelo médico } \\
\text { diarista. }\end{array}$ & $\begin{array}{l}\text { - A falta de adequada } \\
\text { comunicação entre a equipe, } \\
\text { com ausência de registros em } \\
\text { prontuário e opiniões } \\
\text { divergentes em relação à } \\
\text { paliação, foi visualizada como } \\
\text { um obstáculo no } \\
\text { desenvolvimento dos CP na } \\
\text { UTI; } \\
\text { - Faz se necessário a ampliação } \\
\text { das discussões sobre a temática } \\
\text { no cenário da medicina } \\
\text { intensiva, envolvendo o } \\
\text { paciente/família/equipe; } \\
\text { - Necessidade de uma } \\
\text { educação permanente e } \\
\text { continuada da equipe } \\
\text { multidisciplinar sobre cuidados } \\
\text { paliativos em UTI; } \\
\text { - Criação de protocolos } \\
\text { assistenciais para promoção do } \\
\text { conforto do paciente em fase } \\
\text { final da vida e de sua família. }\end{array}$ \\
\hline $\begin{array}{l}\text { Gulini et } \\
\text { al. (2017) }\end{array}$ & $\begin{array}{l}\text { Pesquisa } \\
\text { descritiva, } \\
\text { qualitativa do } \\
\text { tipo } \\
\text { Convergente } \\
\text { Assistencial. }\end{array}$ & $\begin{array}{l}\text { Conhecer a } \\
\text { percepção dos } \\
\text { profissionais de } \\
\text { saúde de uma UTI } \\
\text { acerca do CP }\end{array}$ & $\begin{array}{l}\text { - Observou-se uma falta de } \\
\text { consenso entre os profissionais } \\
\text { de saúde sobre a fase da vida } \\
\text { dirigida para CP; } \\
\text {-A ausência de protocolos } \\
\text { assistenciais dificulta a tomada } \\
\text { de decisão dos cuidados a } \\
\text { serem instituídos e de quais } \\
\text { terapêuticas devem ser } \\
\text { mantidas nesses pacientes; } \\
\text { - Os profissionais de UTI não } \\
\text { estão preparados para lidar com } \\
\text { o paciente em CP, falta } \\
\text { conhecimento, formação } \\
\text { adequada e envolvimento de } \\
\text { todos os integrantes da equipe } \\
\text { multiprofissional. }\end{array}$ & $\begin{array}{l}\text { - A equipe da UTI tem } \\
\text { o entendimento de que } \\
\text { o CP é apropriado na } \\
\text { fase final da vida, sem } \\
\text { necessidade de medidas } \\
\text { fúteis de tratamento, e } \\
\text { com cuidado de } \\
\text { conforto ao paciente e a } \\
\text { seus familiares; }\end{array}$ & $\begin{array}{l}\text { - Necessidade de melhorar a } \\
\text { comunicação entre os } \\
\text { profissionais da equipe da UTI, } \\
\text { para que se estabeleçam } \\
\text { condutas mais uniformizadas } \\
\text { na assistência ao paciente; } \\
\text { - Necessidade de capacitação } \\
\text { para o profissional para que } \\
\text { seja aprimorada a assistência } \\
\text { ao paciente em fase final de } \\
\text { vida; } \\
\text { - Necessidade de construção de } \\
\text { protocolos assistenciais para os } \\
\text { pacientes em CP. }\end{array}$ \\
\hline $\begin{array}{c}\text { Souza et } \\
\text { al. }(2017)\end{array}$ & $\begin{array}{l}\text { Pesquisa } \\
\text { qualitativa, } \\
\text { descritiva. }\end{array}$ & $\begin{array}{l}\text { Compreender o } \\
\text { significado de CP } \\
\text { pela equipe } \\
\text { multiprofissional. }\end{array}$ & $\begin{array}{l}\text { - Entre as dificuldades listadas } \\
\text { pelos participantes na } \\
\text { implementação desses cuidados } \\
\text { estão: o trabalho não } \\
\text { compartilhado em equipe; o } \\
\text { pouco tempo de atuação em } \\
\text { terapia intensiva; o perfil da } \\
\text { UTI; conflitos éticos e ausência } \\
\text { de um protocolo específico; } \\
\text { - Os cuidados devem ser } \\
\text { realizados também com a } \\
\text { família e deve ser respeitada a } \\
\text { autonomia do paciente. }\end{array}$ & $\begin{array}{l}\text { - Os profissionais } \\
\text { possuem o } \\
\text { conhecimento } \\
\text { adequado sobre a } \\
\text { definição dos CP, em } \\
\text { seus aspectos físicos, } \\
\text { emocionais, } \\
\text { psicológicos e } \\
\text { espirituais, quanto da } \\
\text { importância da equipe } \\
\text { multiprofissional. }\end{array}$ & $\begin{array}{l}\text { - Sugere-se a criação de um } \\
\text { protocolo na UTI e de um } \\
\text { momento de discussão de casos } \\
\text { clínicos, incentivando a } \\
\text { atualização da equipe; } \\
\text { - Implementação dos CP nas } \\
\text { grades curriculares dos cursos } \\
\text { de saúde, mudanças nos } \\
\text { códigos de ética e uma maior } \\
\text { discussão sobre o assunto nas } \\
\text { instituições de saúde, desde a } \\
\text { atenção primária, até a } \\
\text { terciária. }\end{array}$ \\
\hline $\begin{array}{l}\text { Mun et al. } \\
\text { (2018) }\end{array}$ & $\begin{array}{l}\text { Pesquisa } \\
\text { descritiva, } \\
\text { qualitativa. }\end{array}$ & $\begin{array}{l}\text { Melhorar os } \\
\text { cuidados em fim de } \\
\text { vida na UTI, } \\
\text { incorporando } \\
\text { processos básicos } \\
\text { de CP no fluxo de } \\
\text { trabalho diário da } \\
\text { UTI. }\end{array}$ & $\begin{array}{l}\text { - Muitas vezes os esforços de } \\
\text { CP realizados pela equipe da } \\
\text { UTI eram ineficazes. }\end{array}$ & $\begin{array}{l}\text { - A identificação } \\
\text { precoce de metas de } \\
\text { atendimento, e as } \\
\text { diretrizes antecipadas } \\
\text { pela equipe da UTI } \\
\text { levou a uma reunião } \\
\text { pró-ativa da família na } \\
\text { UTI; } \\
\text { - As equipes de CP } \\
\text { demonstraram ser } \\
\text { fundamentais na } \\
\text { identificação precoce }\end{array}$ & $\begin{array}{l}\text { - A incorporação dos processos } \\
\text { de CP no fluxo de trabalho } \\
\text { diário da UTI permitiu o } \\
\text { planejamento de cuidados } \\
\text { avançados fossem identificados } \\
\text { de maneira sistemática e pró- } \\
\text { ativa; } \\
\text { - Espera-se que o protocolo } \\
\text { possa levar a mudanças } \\
\text { potenciais no tratamento em } \\
\text { tempo hábil para pacientes } \\
\text { apropriados e melhorar a }\end{array}$ \\
\hline
\end{tabular}




\begin{tabular}{|c|c|c|c|c|c|}
\hline & & & & $\begin{array}{l}\text { de múltiplos aspectos } \\
\text { do planejamento } \\
\text { avançado dos cuidados. }\end{array}$ & $\begin{array}{l}\text { qualidade de vida dos pacientes } \\
\text { e suas famílias que enfrentam } \\
\text { necessidades associadas a } \\
\text { doenças potencialmente fatais. }\end{array}$ \\
\hline $\begin{array}{c}\text { Junior } e t \\
\text { al. (2019) }\end{array}$ & $\begin{array}{l}\text { Pesquisa } \\
\text { descritiva, } \\
\text { qualitativa. }\end{array}$ & $\begin{array}{l}\text { Analisar a } \\
\text { percepção dos } \\
\text { profissionais da } \\
\text { equipe } \\
\text { multidisciplinar } \\
\text { acerca da } \\
\text { assistência em CP. }\end{array}$ & $\begin{array}{l}\text { - Observou-se conhecimento } \\
\text { parcial dos profissionais em } \\
\mathrm{CP} \text {, expressando uma carência } \\
\text { de aprofundamento em relação } \\
\text { a temática; } \\
\text { - Os profissionais relatam o } \\
\text { despreparo em lidar com a } \\
\text { morte e a dificuldade na } \\
\text { implementação da abordagem } \\
\text { paliativista dentro da unidade; } \\
\text { - Necessidade de um } \\
\text { comprometimento efetivo para } \\
\text { a qualidade desse cuidado. }\end{array}$ & $\begin{array}{l}\text {-Os profissionais } \\
\text { reconhecem a } \\
\text { necessidade de oferecer } \\
\text { conforto nos momentos } \\
\text { finais de vida. }\end{array}$ & $\begin{array}{l}\text { - O conhecimento acerca do CP } \\
\text { é limitado, o que leva os } \\
\text { profissionais a vivenciar } \\
\text { dilemas ao lidar com equipe, } \\
\text { paciente e família; } \\
\text { - Dificuldade na } \\
\text { implementação de CP devido à } \\
\text { inaptidão dos profissionais; } \\
\text { - Necessidade do } \\
\text { fortalecimento da } \\
\text { comunicação, do trabalho em } \\
\text { equipe e de um espaço para } \\
\text { discutirem a terminalidade. }\end{array}$ \\
\hline $\begin{array}{c}\text { Baptista e } \\
\text { Picanço } \\
\text { (2019) }\end{array}$ & $\begin{array}{l}\text { Pesquisa } \\
\text { exploratória, } \\
\text { descritiva, } \\
\text { qualitativa. }\end{array}$ & $\begin{array}{l}\text { Conhecer os } \\
\text { saberes da equipe } \\
\text { multiprofissional } \\
\text { sobre CP em UTI. }\end{array}$ & $\begin{array}{l}\text { - Dificuldade de compreensão } \\
\text { do melhor momento para início } \\
\text { dos CP; } \\
\text { - Desconhecimento dos } \\
\text { conceitos preconizados sobre } \\
\text { CP, como quando se deve } \\
\text { iniciar, as fases que compõe, } \\
\text { além da falta de entendimento } \\
\text { sobre o significado de CP } \\
\text { exclusivos. }\end{array}$ & $\begin{array}{l}\text { - Observou-se a } \\
\text { preocupação da equipe } \\
\text { com o alívio dos } \\
\text { sintomas físicos } \\
\text { proporcionando o } \\
\text { conforto e diminuição } \\
\text { do sofrimento } \\
\text { psicossocial; } \\
\text { - Constatou-se a } \\
\text { inclusão da família } \\
\text { como parte do cuidado } \\
\text { e as necessidades de } \\
\text { garantir qualidade de } \\
\text { vida. }\end{array}$ & $\begin{array}{l}\text { - A maioria dos profissionais } \\
\text { de saúde reconhece a } \\
\text { necessidade de qualidade de } \\
\text { vida e conforto diante do } \\
\text { paciente que tem uma doença } \\
\text { que ameace a vida; } \\
\text { - O trabalho profissional com } \\
\text { pacientes com doença que } \\
\text { ameace a vida exige formação } \\
\text { especial, incluindo capacitação } \\
\text { e atualização contínua sobre o } \\
\text { assunto. }\end{array}$ \\
\hline $\begin{array}{l}\text { Pegoraro e } \\
\text { Paganini } \\
(2019)\end{array}$ & $\begin{array}{l}\text { Pesquisa } \\
\text { qualitativa. }\end{array}$ & $\begin{array}{l}\text { Investigar o } \\
\text { conhecimento da } \\
\text { equipe e elaborar } \\
\text { ações de CP a } \\
\text { pacientes com } \\
\text { limitação de } \\
\text { suporte de vida em } \\
\text { UTI. }\end{array}$ & $\begin{array}{l}\text { - Posicionamento tímido dos } \\
\text { profissionais quanto a } \\
\text { proporcionar morte digna e sem } \\
\text { sofrimento para os pacientes } \\
\text { que morrem em UTI com } \\
\text { doenças consideradas terminais; } \\
\text { - A grande complexidade } \\
\text { existente entre duas concepções } \\
\text { ainda em construção no cenário } \\
\text { brasileiro: CP X Cuidados } \\
\text { intensivos. }\end{array}$ & $\begin{array}{l}\text { - A equipe } \\
\text { multidisciplinar } \\
\text { demonstrou entender e } \\
\text { ser capaz de identificar } \\
\text { assuntos relacionados } \\
\text { ao tema; } \\
\text { - Os profissionais } \\
\text { acreditam oferecer } \\
\text { morte digna, evitar } \\
\text { distanásia e não } \\
\text { prolongar o sofrimento } \\
\text { do paciente; } \\
\text { - Verificou-se que os } \\
\text { profissionais entendiam } \\
\text { os CP como abordagem } \\
\text { que envolve equipe } \\
\text { multiprofissional, que } \\
\text { deve assistir não só ao } \\
\text { paciente, mas também } \\
\text { à sua família. }\end{array}$ & $\begin{array}{l}\text { - Os profissionais de saúde } \\
\text { reconheceram a necessidade de } \\
\text { estabelecer critérios para } \\
\text { atender pacientes com } \\
\text { limitação de suporte de vida } \\
\text { em UTI e a importância dos } \\
\text { CP, sendo possível aplicá-los } \\
\text { por meio de ações } \\
\text { sistematizadas; } \\
\text { - As instituições devem } \\
\text { proporcionar momentos de } \\
\text { análise e reflexão sobre o } \\
\text { processo de trabalho dos } \\
\text { profissionais, com olhar mais } \\
\text { atento ao CP de pacientes em } \\
\text { limitação de suporte de vida. }\end{array}$ \\
\hline $\begin{array}{l}\text { Kyereman } \\
\text { teng } \text { et al. } \\
\text { (2019) }\end{array}$ & $\begin{array}{l}\text { Pesquisa } \\
\text { descritiva, } \\
\text { qualitativa. }\end{array}$ & $\begin{array}{l}\text { Avaliar a qualidade } \\
\text { percebida na } \\
\text { melhora dos } \\
\text { cuidados de final } \\
\text { de vida nas } \\
\text { instituições } \\
\text { participantes e } \\
\text { explorar barreiras } \\
\text { para a integração } \\
\text { UTI-CP. }\end{array}$ & $\begin{array}{l}\text { - Há uma presença deficiente de } \\
\text { CP na UTI; } \\
\text { - A principal barreira à } \\
\text { integração são as expectativas } \\
\text { irreais dos pacientes e / ou } \\
\text { familiares. }\end{array}$ & $\begin{array}{l}\text { - A maioria dos } \\
\text { entrevistados percebe } \\
\text { que a presença de CP } \\
\text { na UTI pode melhorar } \\
\text { os cuidados de final de } \\
\text { vida. }\end{array}$ & $\begin{array}{l}\text { - O aumento da integração } \\
\text { UTI-CP pode melhorar a } \\
\text { qualidade dos cuidados de final } \\
\text { de vida; } \\
\text { - Iniciativas futuras de } \\
\text { melhoria da qualidade podem } \\
\text { se concentrar no } \\
\text { desenvolvimento de um } \\
\text { conjunto de critérios para } \\
\text { acionar consultas de CP. }\end{array}$ \\
\hline $\begin{array}{l}\text { Pires et al. } \\
\text { (2020) }\end{array}$ & $\begin{array}{l}\text { Pesquisa } \\
\text { qualitativa, } \\
\text { descritiva e } \\
\text { exploratória. }\end{array}$ & $\begin{array}{l}\text { Analisar a } \\
\text { percepção da } \\
\text { equipe } \\
\text { multiprofissional } \\
\text { sobre o conforto no } \\
\text { final de vida em } \\
\text { UTI. }\end{array}$ & $\begin{array}{l}\text { - Os profissionais } \\
\text { demonstraram utilizar práticas } \\
\text { assistenciais para promoção de } \\
\text { um fim de vida pacífico que } \\
\text { não estão padronizados em } \\
\text { protocolos. }\end{array}$ & $\begin{array}{l}\text { - O conforto foi o } \\
\text { conceito da que se } \\
\text { destacou na percepção } \\
\text { da equipe } \\
\text { multiprofissional, } \\
\text { sendo promovido por } \\
\text { todas as categorias na }\end{array}$ & $\begin{array}{l}\text { - A percepção da equipe } \\
\text { multiprofissional sobre CP } \\
\text { corrobora com os conceitos da } \\
\text { Teoria do Final de Vida } \\
\text { Pacífico, sugerindo a } \\
\text { identificação das necessidades } \\
\text { básicas de cada paciente e }\end{array}$ \\
\hline
\end{tabular}




\begin{tabular}{|c|c|c|c|c|c|}
\hline & & & & $\begin{array}{l}\text { sua prática assistencial } \\
\text { a pacientes em } \\
\text { paliação; } \\
\text { - A suspensão de } \\
\text { procedimentos para } \\
\text { melhorar o conforto do } \\
\text { paciente em CP e evitar } \\
\text { intervenções dolorosas; } \\
\text { - A aproximação dos } \\
\text { familiares durante a } \\
\text { internação foi } \\
\text { percebida como } \\
\text { mecanismo de redução } \\
\text { de fatores ambientais } \\
\text { hostis. }\end{array}$ & $\begin{array}{l}\text { discussão multiprofissional } \\
\text { para construção de um plano de } \\
\text { cuidados, direcionado para a } \\
\text { promoção do conforto; } \\
\text { - Sinalização da necessidade de } \\
\text { capacitação que desperte a } \\
\text { promoção de forma } \\
\text { multidimensional; } \\
\text { - Criar estratégias de } \\
\text { intervenção para o alivio da } \\
\text { dor, aproximação com entes } \\
\text { queridos, promoção de } \\
\text { paz/dignidade/respeito e } \\
\text { valorização da fé são } \\
\text { dimensões do cuidado que } \\
\text { possibilitam um final de vida } \\
\text { pacífico e que poderão ser } \\
\text { utilizadas como protocolo de } \\
\text { atuação da equipe em estudos } \\
\text { futuros. }\end{array}$ \\
\hline $\begin{array}{l}\text { Maingué } \\
\text { et al. } \\
\text { (2020) }\end{array}$ & $\begin{array}{l}\text { Pesquisa } \\
\text { exploratória, } \\
\text { descritiva e } \\
\text { quantitativa. }\end{array}$ & $\begin{array}{l}\text { Identificar fatores } \\
\text { que influenciam a } \\
\text { tomada de decisões } \\
\text { de profissionais de } \\
\text { saúde diante de } \\
\text { pacientes em } \\
\text { cuidados de fim de } \\
\text { vida internados em } \\
\text { UTI. }\end{array}$ & $\begin{array}{l}\text { - Conflitos entre a equipe } \\
\text { multiprofissional são } \\
\text { frequentes, e a maior parte } \\
\text { envolve divergências sobre } \\
\text { prognóstico e plano terapêutico; } \\
\text { - Questões relacionadas à } \\
\text { terminalidade da vida } \\
\text { costumam ser foco de } \\
\text { desentendimento e desgaste } \\
\text { entre os profissionais, afetando } \\
\text { a assistência e muitas vezes } \\
\text { acarretando demora na tomada } \\
\text { de decisão. }\end{array}$ & $\begin{array}{l}\text { - Decisões } \\
\text { compartilhadas } \\
\text { repercutem } \\
\text { positivamente na } \\
\text { assistência; } \\
\text { - Discussões sobre o } \\
\text { tratamento de doentes } \\
\text { terminais são regulares } \\
\text { dentro das equipes } \\
\text { multiprofissionais, e } \\
\text { poderiam até ser mais } \\
\text { frequentes, pois } \\
\text { aumentam o } \\
\text { conhecimento e } \\
\text { diminuem os conflitos. }\end{array}$ & $\begin{array}{l}\text { - Constatou-se preocupação } \\
\text { dos entrevistados em respeitar } \\
\text { a autonomia, proteger a } \\
\text { dignidade e preservar a } \\
\text { qualidade de vida de pacientes } \\
\text { e familiares por meio da } \\
\text { decisão compartilhada; } \\
\text { - Tendência de obstinação } \\
\text { terapêutica para cumprir o } \\
\text { dever profissional mostrou } \\
\text { necessidade de mais discussões } \\
\text { e formação em CP para } \\
\text { minimizar conflitos éticos. }\end{array}$ \\
\hline $\begin{array}{l}\text { Pavlish et } \\
\text { al. (2020) }\end{array}$ & $\begin{array}{l}\text { Pesquisa } \\
\text { descritiva de } \\
\text { pré e pós } \\
\text { intervenção. }\end{array}$ & $\begin{array}{l}\text { Investigar a } \\
\text { eficácia de um } \\
\text { protocolo de ética } \\
\text { pró-ativo e baseado } \\
\text { em equipe. }\end{array}$ & $\begin{array}{l}\text { - Os conflitos éticos complicam } \\
\text { a prática clínica e } \\
\text { comprometem a comunicação e } \\
\text { o trabalho em equipe entre } \\
\text { pacientes, familiares e clínicos; } \\
\text { - O tempo de permanência na } \\
\text { UTI não mudou. }\end{array}$ & $\begin{array}{l}\text { - Quando as equipes de } \\
\text { saúde foram } \\
\text { incentivadas a se } \\
\text { comunicar } \\
\text { rotineiramente sobre as } \\
\text { metas de atendimento, } \\
\text { mais pacientes } \\
\text { receberam o apoio } \\
\text { necessário e as } \\
\text { barreiras à } \\
\text { comunicação foram } \\
\text { reduzidas. }\end{array}$ & $\begin{array}{l}\text { - As chances de receber uma } \\
\text { conferência familiar e uma } \\
\text { visita religiosa foram } \\
\text { significativamente maiores } \\
\text { após a intervenção; } \\
\text { - O número de consultas sobre } \\
\text { CP e discussões de tratamento } \\
\text { aumentou em } 3 \text { e } 6 \text { meses. }\end{array}$ \\
\hline
\end{tabular}

Fonte: Autores.

A partir dos dados levantados nos estudos selecionados para essa revisão, foi possível identificar que a maioria das pesquisas foram de natureza descritiva e qualitativa. Os membros da equipe multiprofissional eram principalmente enfermeiros, técnicos de enfermagem, fisioterapeutas e médicos. Entre as fragilidades encontradas, destacam-se a falta de interação e comunicação entre os profissionais da equipe multidisciplinar, conflitos éticos e ausência de um protocolo específico, desprepraro dos profissionais de UTI para lidar com o paciente em CP, falta conhecimento, formação adequada e envolvimento de todos os integrantes da equipe. E as potencialidades mais observadas foram de que a equipe da UTI tem o entendimento de que o CP é apropriado na fase final da vida, sem necessidade de medidas fúteis de tratamento, e com cuidado de conforto ao paciente e a seus familiares. 


\section{Discussão}

A proposta de discussão do objeto deste estudo pode evidenciar potencialidades e fragilidades que estão relacionadas à assistência no CP pela equipe multiprofissional dentro das UTIs, as quais podem subsidiar ações institucionais mais específicas para o maior conhecimento sobre esse cuidado e melhores estratégias para sua implementação.

Observa-se que apesar de ainda estar se desenvolvendo em todo o mundo, os CP estão progressivamente integrandose aos cuidados curativos, inclusive nos ambientes de terapia intensiva (Gulini et al., 2017). A limitação de suporte de vida tem sido aplicada na terapia intensiva em quadros de doença irreversível e tratamento improdutivo (Pavlish et al., 2020). Nessas situações, cuidados paliativos visam prevenir e aliviar o sofrimento e tornam-se essenciais para propiciar atenção específica e contínua para o paciente e sua família, possibilitando morte digna (Pegoraro \& Paganini, 2019).

O CP é uma abordagem que promove a qualidade de vida de pacientes fora da possibilidade de tratamento modificador da doença. Voltado para medidas de conforto, o CP dá-se por meio da prevenção e alívio do sofrimento e da assistência à família no enfrentamento de dificuldades associadas à doença com risco de morte (Junior et al., 2019). Nesse sentido, a identificação precoce, avaliação e tratamento da dor e outras adversidades de natureza física, psicossocial e espiritual são essenciais na abordagem paliativista. O excelente controle de sintomas, compromisso contínuo de atenção ao paciente e a família, o apoio físico, psicológico e espiritual são as marcas dos cuidados de alta qualidade no fim da vida (Silva et al., 2013). Esse cuidado está emergindo na UTI e exige o mesmo nível elevado de conhecimento e competência de todas as outras áreas envolvidas. Entretanto, várias evidências têm demonstrado que esta abordagem ainda precisa melhorar, seja para os pacientes com sintomas de desconforto significativos ou dores físicas, nas UTI, ou na percepção dos familiares, que também é falha (Gulini et al., 2017).

Diante do processo de morrer, as UTIs são associadas à dor e ao sofrimento de pacientes e suas famílias (Maingué et al., 2020). O uso de tecnologias complexas, a pouca comunicação entre profissionais, paciente e familiares, a gravidade dos casos clínicos e, principalmente, a proximidade com a morte são fatores que acentuam essa relação (Souza et al., 2017). Assim, esse mesmo autor enfatiza a necessidade de rever o modo como decisões são apresentadas a pacientes e familiares, a fim de minimizar o receio e a dúvida quanto à recuperação da qualidade de vida e da saúde (Souza et al., 2017). Isso requer mudanças estruturais que priorizem a comunicação entre os envolvidos, criando condutas para tornar o processo de morrer menos angustiante para todos (Pegoraro \& Paganini, 2019). Nesse sentido, a estratégia de integrar CP à prestação de cuidados intensivos tem renovado esta área (Maingué et al., 2020).

Segundo Junior et al. (2019), o paliativismo deve envolver uma equipe multiprofissional, incluindo médicos, enfermeiros, fisioterapeutas, psicólogos, psiquiatras, nutricionistas, fonoaudiólogos, assistentes sociais, farmacêuticos e conselheiros espirituais, para tornar o cuidado mais integrado e fornecer ao paciente crítico toda a oportunidade de cuidado existente, garantindo, assim, uma melhor qualidade de vida.

Nesse sentindo, a equipe multiprofissional vivencia uma mudança do paradigma do cuidado, buscando melhorar a qualidade de vida daqueles que enfrentam problemas associados a doenças ameaçadoras de vida, envolvendo o cuidado do sofrimento para além dos sintomas físicos, o que inclui o apoio de uma equipe multidisciplinar e o atendimento das necessidades básicas do paciente e sua família (Pires et al., 2020).

Alguns estudos relatam que os membros da equipe multiprofissional das UTIs demonstram uma compreensão adequada, de um modo geral, do significado de CP (Gulini et al., 2017; Souza et al., 2017; Mun et al., 2018; Kyeremanten et al., 2019; Pegoraro \& Paganini, 2019). Porém outros autores trazem que a maioria dos profissionais de saúde reconhece a necessidade de qualidade de vida e conforto diante do paciente que tem uma doença que ameace a vida. No entanto, desconhecem os conceitos preconizados sobre $\mathrm{CP}$ e algumas premissas, como quando se deve iniciar, as fases que compõe, além da falta de entendimento sobre o significado dos CP exclusivos (Silva et al., 2013; Baptista \& Picanço, 2019; Junior et 
al., 2019). Isto expressa uma carência de aprofundamento em relação a temática, e despreparo em lidar com a morte, assim como a dificuldade na implementação da abordagem paliativista dentro da unidade.

Alguns estudos selecionados para compor a revisão relatam que uma adequada comunicação entre todos os envolvidos é fundamental, e que a dificuldade de comunicação entre os profissionais de saúde, os pacientes e familiares é sem dúvida uma das principais barreiras geradoras de conflitos na assistência ao paciente crítico sem possibilidades terapêuticas (Silva et al., 2013; Gulini et al., 2017; Souza et al., 2017; Pavilish et al., 2020).

Além das dificuldades de comunicação entre a equipe, outro obstáculo encontrado no desenvolvimento dos CP foi a ausência de registros em prontuário e opiniões divergentes em relação à paliação (Souza et al., 2017). A comunicação é um desafio nas UTIs devido a múltiplos aspectos fundamentais dos cuidados intensivos, sendo um deles a complexidade (Maingué et al., 2020). Foi relatado também a incerteza como uma constante no que diz respeito aos resultados, afetando a tomada de decisão e acrescentando estresse para todos os envolvidos, incluindo pacientes, famílias e equipe de saúde (Pavilish et al., 2020).

Assim Junior et al. (2019) complementam dizendo que aos profissionais da equipe de saúde recomenda-se o uso da comunicação honesta e completa, porém sensível à escuta do outro. E ainda que o enfrentamento da doença por parte do paciente e familiares poderá ser concretizada pelo estabelecimento de uma comunicação de qualidade, que resulte na confiança e participação na tomada de decisões.

Outro ponto negativo observado é a percepção de que na UTI os profissionais não estão preparados para lidar com o paciente em CP, seja por falta de conhecimento, de formação adequada e envolvimento de todos os integrantes da equipe multiprofissional (Souza et al., 2017). Embora a UTI seja um local em que a morte está sempre presente, os profissionais que prestam os cuidados intensivos não estão adequadamente treinados ou qualificados para o atendimento de fim de vida (Gulini et al., 2017).

Portanto, essa equipe deve ser reconhecida como provedora, mas também como objeto dos cuidados. O trabalho profissional com pacientes com doença que ameace a vida exige formação especial, incluindo capacitação e atualização contínua sobre o assunto, e é por meio da convivência com esse tema que os profissionais se tornarão cada vez mais habilitados e confiantes na assistência prestada aos pacientes sob seus cuidados (Baptista \& Picanço, 2019).

A educação entre os profissionais de saúde, desde sua formação, com apropriada informação e treinamento torna-se fundamental, sendo de suma importância que sejam oferecidos treinamento e educação continuada, que capacite esses profissionais, de modo permanente, para os CP (Kyeremanten et al., 2019).

Definir quais cuidados deverão ser mantidos e quais deverão ser suspensos na abordagem paliativista também é uma das decisões mais difíceis de serem tomadas pela equipe (Silva et al., 2013). Devido à ausência de protocolos, escassez de conhecimento sobre a temática e sobre os aspectos ético-legais envolvidos, a equipe encontra dificuldade em indicar a abordagem paliativa e determinar os cuidados a serem efetuados (Souza et al., 2017). Assim, a criação de protocolos específicos, ainda geralmente inexistentes nas UTIs, pode contribuir para direcionar os cuidados a serem executados, e servir de guia para a equipe multiprofissional, buscando diminuir o sofrimento do paciente e de sua família, e desta forma promover uma morte digna e tranquila (Silva et al., 2013; Gulini et al., 2017; Souza et al., 2017; Mun et al., 2018; Pires et al., 2020).

\section{Considerações Finais}

A partir dos resultados encontrados, pode-se observar a importância de se ter uma equipe multiprofissional especializada no atendimento de pacientes sem possibilidade de cura em todas as dimensões, garantindo-lhes o bem-estar e respeito à sua dignidade. É imperativo que o CP se integre ao ambiente da UTI e se consolide como uma filosofia de cuidado que busca prover o alívio da dor e de outros sintomas, dando um suporte espiritual e psicossocial ao fim da vida e ao luto. 
Enfatizando, por fim, que esses profissionais ainda não possuem um total entendimento sobre os CP, e nem são capacitados para isso. Por isso, é necessário melhorar a comunicação entre os profissionais da equipe para que se estabeleçam condutas mais uniformizadas na assistência ao paciente, como a capacitação destes para que em conjunto seja aprimorada a assistência ao paciente em fase final de vida, e oferecer o suporte necessário aos familiares.

A educação entre os profissionais de saúde, desde sua formação, com apropriada informação e treinamento torna-se fundamental, sendo de suma importância que sejam oferecidos treinamento e educação continuada, que capacite esses profissionais, de modo permanente para os $\mathrm{CP}$.

Além disso, a implantação de protocolos específicos em cuidados paliativos nos ambientes de terapia intensiva poderá reduzir o sofrimento e melhorar a qualidade do atendimento oferecido ao paciente em fase final de vida. Estes poderão servir de guia para a equipe multiprofissional, ajudando a esclarecer, descrever e obter consenso sobre as normas para fim de vida na tomada de decisão e cuidado, e assim melhorar a satisfação com a colaboração multiprofissional e assistência ao paciente como um todo.

\section{Referências}

Baptista, S. C. O \& Picanço, C. M. (2019). Cuidados paliativos em unidade de atendimento crítico: saberes de uma equipe multiprofissional. Enfermagem Brasil, 18 (5), 612-624.

Boyle, D. K, Miller, P. A \& Forbes-Thompson, S. A. (2005). Communication and End-of-Life Care in the Intensive Care Unit: Patient, Family, and Clinician Outcomes. Critical Care Nursing Quarterly, 28 (4), 302-316.

Byock, I. M. D. (2006). Where do we go from here? A palliative care perspective. Critical Care Medicine, 34 (11), $416-420$.

Clemens, K. E. \& Klaschik, E. (2009). Integration of principles of palliative medicine into treatment of patients in intensive care units. Anasthesiologie, Intensivmedizin, Notfallmedizin, Schmerztherapie: AINS, 44 (2), 88-94.

Coelho, C. B. T. \& Yankaskas, J. R. (2017). Novos conceitos em cuidados paliativos na unidade de terapia intensiva. Revista Brasileira Terapia Intensiva, 29 (2), 222-230.

Gulini, J.E.M.B., Nascimento, E. R. P., Moritz. R. D., Rosa, L. M., Silveira, N. R. \& Vargas. M. A. O. (2017). A equipe da Unidade de Terapia Intensiva frente ao cuidado paliativo: discurso do sujeito coletivo*. Revista da Escola de Enfermagem da USP, 5 (1), 1-6.

Ivany, E. \& Aitken, L. (2019). Challenges and facilitators in providing effective end of life care in intensive care units. Nursing Standard, 34 (6), 44-50.

Junior, A. R., Moreira. T. M. M., Florêncio, R. S., Souza, L. C., Flor, A. C. \& Pessoa, V. L. M. P. (2019). Conforto nos momentos finais da vida: a percepção da equipe multidisciplinar sobre cuidados paliativos. Revista Enfermagem UERJ, 27 (45135), 1-6.

Klein, C., Heckel, M., Treibig, T., Hofmann, S., Ritzer-Rudel, I. \& Ostgathe, C. (2012). The palliative care team in the intensive care unit. Medizinische Klinik, Intensivmedizin und Notfallmedizin, 107 (4), 240-243.

Kyeremanteng, K., Beckerleg, W. \& Wan, C. (2020). Survey on Barriers to Critical Care and Palliative Care Integration. American Journal of Hospice and Palliative Care, 37 (2), 108-116.

Maingué, P. C. P. M., Sganzerla, A., Guirro, U. B. P. \& Perini, C. C. (2020). Discussão bioética sobre o paciente em cuidados de fim de vida. Revista Bioética, 28 (1), 135-146.

Moritz, R. D., Machado, F. O., Heerdt, M., Rosso, B. \& Beduschi, G. (2009). Avaliação das decisões médicas durante o processo do morrer. Revista Brasileira de Terapia Intensiva, 21 (2), 141-147.

Mun, E., Umbarger, L., Ceria-Ulep, C. \& Nakatsuka, C. (2018). Palliative Care Processes Embedded in the ICU Workflow May Reserve Palliative Care Teams for Refractory Cases. American Journal of Hospice and Palliative Care, 35 (1), 60-65.

O’Mahony, S., McHenry, J., Blank, A. E., Snow, D., Karakas, S. E., Santoro, G., Selwyn, P. \& Kvetan, V. (2010). Preliminary report of the integration of a palliative care team into an intensive care unit. Palliative Medicine, 24 (2), 154-65.

Pavlish, C. L., Henriksen, J., Saltzman, K. B., Robinson, E. M., Warda, U. S., Farra, C., Chen, B. \& Jakel, P. (2020). A Team-Based Early Action Protocol to Address Ethical Concerns in the Intensive Care Unit. American Journal of Critical Care, 29, (1), 49-61.

Pegoraro, M. M. O. \& Paganini, M. C. (2019). Cuidados paliativos e limitação de suporte de vida em terapia intensiva. Revista Bioética, 27 (4), 699-710.

Pires, I. B., Menezes, T. M. O., Cerqueira, B. B., Albuquerque, R. S., Moura, H. C. G. B., Freitas, R. A., Santos, A. L. S. \& Oliveira, E. S. (2020). Conforto no final de vida na terapia intensiva: percepção da equipe multiprofissional. Acta Paulista de Enfermagem, 33, 1-7.

Santana, J. C. B., Dutra, B. S., Carlos, J. M. M. \& Barros, J. K. A. (2017). Ortotanásia nas unidades de terapia intensiva: percepção dos enfermeiros. Revista Bioética, 25 (1), 158-167. 
Research, Society and Development, v. 10, n. 9, e22210917989, 2021

(CC BY 4.0) | ISSN 2525-3409 | DOI: http://dx.doi.org/10.33448/rsd-v10i9.17989

Santos, D. C. L., Silva, M. M., Moreira, M. C., Zepeda, K. G. M. \& Gaspar. R. B. (2017). Planejamento da assistência ao paciente em cuidados paliativos na terapia intensiva oncológica. Acta Paulista de Enfermagem, 30 (3), 295-300.

Santos, F. S. (2011). Cuidados paliativos: diretrizes, humanização e alívio de sintomas, Atheneu, 654.

Silva, C. F., Souza, D. M., Pedreira, L. C., Santos, M. R. \& Faustino, T. N. (2013). Concepções da equipe multiprofissional sobre a implementação dos cuidados paliativos na unidade de terapia intensiva. Ciência \& Saúde Coletiva, 18 (9), 2597-2604.

Souza, H. L. R., Andrade e Lacerda, L. C. \& Lira, G. G. (2017). Significado de Cuidados Paliativos pela Equipe Multiprofissional da Unidade de Terapia Intensiva. Revista de enfermagem UFPE online, 11(10), 3885-3892.

Vicensi, M. C. (2016). Reflexão sobre a morte e o morrer na UTI: a perspectiva do profissional. Revista Bioética, 24 (1), 64-72. 